\title{
BER Analysis of WiMAX using Diverse Channel Encoding Schemes over AWGN and Rayleigh Channel
}

\author{
Mahwash Manzoor and Lavish Kansal \\ School of Electronics and Electrical Engineering, Lovely Professional University, Punjab - 144411, India; \\ mahwash25@yahoo.com, lavish.15911@lpu.co.in
}

\begin{abstract}
WiMAX is the modern era wireless technology which gained popularity because of its efficiency, high coverage, scalability and high data rates in any terrain. WiMAX is a Wireless Metropolitan Area Network (WMAN) being used globally for broadband access. There is always a demand for improvement in systems, which in the case of WiMAX can be achieved by using a better coding scheme with a proper modulation technique. In this paper, a comparison has been made on performances of different forward error correction codes on two channels- AWGN and Rayleigh channel. AWGN channel simply adds white Gaussian noise to the signal transmitted through it whereas Rayleigh channel is a fading multipath channel whose characteristics vary continuously with time. The results show that Turbo codes perform the best amongst all the coding techniques, and AWGN channel response is much better than the response of Rayleigh channel. This is because there is no line of sight component in Rayleigh channel and having obstructions in the path of the signal leads to signal fading. These results are shown in this paper as graphical analysis between BER and SNR.
\end{abstract}

Keywords: Additive White Gaussian Noise (AWGN), Bit Error Rate (BER), Signal to Noise Ratio (SNR), Wireless Metropolitan Area Network (WMAN), Worldwide Interoperability for Microwave Access (WiMAX)

\section{Introduction}

WiMAX stands for Worldwide Interoperability for Microwave Access. It's a rapidly growing technology which works like any other mobile communication system. Like any mobile communication system, it also has its base station (BS) and a mobile station (MS). The mobile station can operate only within the range of the base station 1 . WiMAX is a diverse technology that combines many technologies like interoperability, FDD, TDD, OFDMA, and ARQ, etc. Interoperability as in it can be operated with previous generations of the system resulting in cost reduction. FDD and TDD are the duplexing techniques being used in WiMAX, mostly they are used in combination. OFDMA is the basis of WiMAX and stands for Orthogonal Frequency Division Multiple Access. Its like other multiple access schemes with the difference that the sub-carriers are orthogonal to each other preventing ISI. ARQ is automatic retransmission request which is used in case of failure in the reception of data ${ }^{2}$. OFDM is the most important part of WiMAX system since it gives high data rates by distributing the data onto orthogonal subcarriers which helps to provide high speed and remove ISI. OFDM, when used with some good channel code, provides better performance in frequency selective fading channels. The code to be used should have decent

${ }^{*}$ Author for correspondence 
error detecting and correcting capability and a high coding gain ${ }^{3}$. The channel codes include convolutional codes, RS codes, BCH codes, Turbo codes and now the recently popular LDPC codes. Sometimes, a combination of codes called concatenated or product codes has to be used to improve the performance further. $\mathrm{BCH}$ codes can also be used with WiMAX system to improve its performance. $\mathrm{BCH}$ codes were developed independently by three scientists Bose, R. Chaudhuri and Hocquengham. These codes require a primitive element and a generator polynomial. They can correct a particular number of errors. The famous decoding algorithm for $\mathrm{BCH}$ codes is Berlekamps algorithm $\frac{4,5}{.}$. Reed-Solomon codes are a class of nonbinary $\mathrm{BCH}$ codes which are again a part of cyclic codes. They are used for real-time applications and correction of burst errors. They can be easily used for WMANs and hence can be used in WiMAX technology ${ }^{\frac{6,7}{7} \text {. Turbo codes }}$ are somewhat similar to convolutional codes because they make use of recursive systematic convolutional encoders (RSC) plus interleaver between the two RSCs. It is in a way parallel concatenation of convolutional codes (PCCC). They perform well when implemented with WiMAX systems. When a combination of LDPC-turbo codes is used it performs better than the constituent codes. LDPC codes perform well with high data rates whereas turbo codes perform well with low data rates, so a combination of both exploits the best properties of both the $\operatorname{codes}^{8,9}$. For mobile WiMAX (IEEE-802.16e), the best coding technique now-a-days is LDPC coding. It was developed in the early 1960s but did not gain the required response until recently because of its high complexity in computation and decoding. It got its name from its parity check matrix which is of low density that is, sparse in nature. It has very small number of ones and a large number of zeroes. These codes can achieve near Shannon limit performance. These codes can be concatenated with turbo codes for additional improvement in performance ${ }^{10,11}$. To fulfill the growing demand for high speed and high data rates with large coverage region the last mile option was discovered called broadband wireless access or WiMAX. To improve the performance of this system better channel coding techniques and statistical models of channels have been used, and those are incorporated into a good modulation scheme to achieve efficiently high performance of the same system ${ }^{12}$. This paper contains WiMAX with four different modulation orders of PSK techniques and two different channels namely Rayleigh channel and AWGN channel, and the response is studied for four different channel coding techniques i.e. BCH codes, Turbo codes, LDPC codes and RS codes.

\section{Model Description of Wimax Physical Layer}

The WiMAX physical layer consists of various blocks that work together to lead to the proper working of such a broadband mobile communication system. Like any other communication system WiMAX consists primarily of three parts-transmitter, channel, and receiver. The transmitter and receiver are connected using a wireless or radio channel. It is in the channel that signal gets corrupted by noise and fades away hence causing loss of information. This paper is based on the use of two channels for analysis namely- Rayleigh channel and AWGN channel. The transmitter and receiver consist of five blocks each as shown in Figure 1.

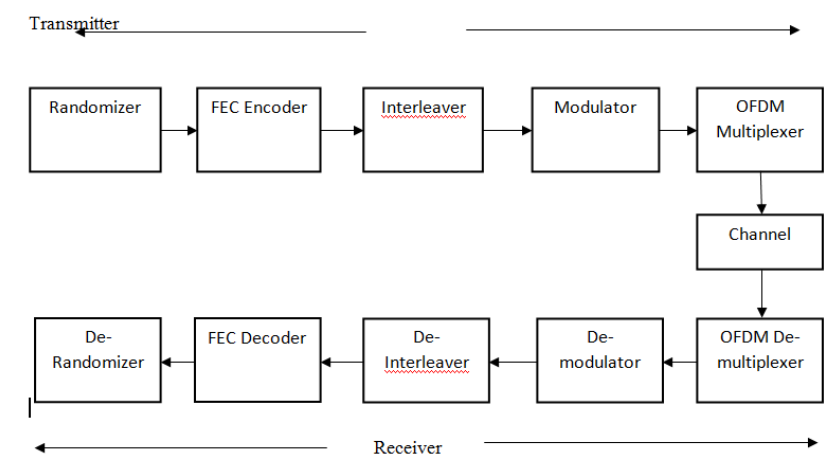

Figure 1. Block diagram of WiMAX Physical Layer.

\subsection{Transmitter}

The transmitter block consists of- randomizer, FEC encoder, interleaver, modulator and OFDM multiplexer. Each of these has a particular function assigned to them and are explained one by one as below.

1. Randomizer is used to arbitrarily change the sequence of data so that there is unpredictability in data and when fading occurs, it equally affects the data or, in other words, the probability of data being lost is equal to all bits or blocks of data. It is implemented using shift registers which bring about randomness in the bits.

2. FEC encoder is used to add redundancy to the data at the transmitter side. It has ' $k$ ' number of bits as an 
input and ' $\mathrm{n}$ ' number of bits as an output, where ' $\mathrm{n}$ ' is always greater than ' $k$. The quantity ' $k / n$ ' is called as code rate of the used code. The reciprocal of code rate gives the amount of redundancy. There are various FEC codes like block codes, cyclic codes, etc. The ones used in this paper are-BCH codes, Turbo codes, LDPC codes and RS codes. Each of these codes has several advantages and disadvantages associated with them and are used to suit a particular application.

3. Interleaver is a block that permutes the data. It is mostly based on two permutations. The most important and commonly used interleaver is block interleaver. In this type of interleaver, we take a matrix representation of interleaver where bits of data are written in a row fashion and read in a column fashion. In this way, deep fading of signals can be recovered because there are no consecutive bits of errors.

4. The modulator is in its primitive sense is a block which changes one or more characteristics of the carrier signal by the information bearing signal. The characteristics of the carried being varied may be the phase, amplitude or frequency. In this paper, digital modulation is used and that too PSK of various modulation orders. The modulator here maps the data bits into signals so that they may be propagated through the channel easily. The order of modulation depends on how many bits are taken simultaneously to form a signal.

5. OFDM multiplexer is the most crucial block of WiMAX system. The signal which is wideband is divided into many narrowband signals which are orthogonal to each other. The condition of orthogonality is of prime importance because it is because of these subcarriers being orthogonal that signals have reduced inter symbol interference (ISI). It also prevents frequency selective fading. The subcarriers are of different frequencies, and guard bands are also added to them to prevent mixing of signals.

\subsection{Channel}

The channels used in mobile communication are radio channels where the air is the medium of propagation. To get the statistical representation of channels, various models have been proposed. Since channels are time variant and random, there nature cannot be predicted at any point of time. The two channels used in this paper are AWGN channel and Rayleigh channel.
1. AWGN channel stands for additive white Gaussian noise channel. This channel only adds white noise to the signal being transmitted through it. White noise has a power spectral density of $\mathrm{N}_{0} / 2$. Mathematically the channel can be represented by:

$$
r=s_{m}+n
$$

Where, ' $r$ ' is the received signal vector, 'Sm' is the transmitted signal vector and ' $n$ ' is the noise vector that corrupts the signal. The probability density function of noise vector is given below:

$$
p(n)=\left(\frac{1}{\sqrt{\Pi N_{0}}}\right)^{N} \exp \left(-\frac{\sum_{k=1}^{N} n_{k}^{2}}{2 \sigma^{2}}\right)
$$

2. Rayleigh channel is a multipath channel in which fading is by Rayleigh distribution. The sum of two Gaussian random variables is always a Rayleigh distribution. It is a multipath channel model where scattering takes place, and the received signal has no line of sight component. It has a time varying nature and is also defined by a probability density function as given below:

$$
p(n)=\left\{\begin{aligned}
\frac{n}{\sigma^{2}} \exp -\frac{n^{2}}{2 \sigma^{2}}, & 0 \leq n \leq \infty \\
0, & n<0
\end{aligned}\right.
$$

\subsection{Receiver}

The blocks on the receiver side are the reciprocal blocks of those on the transmitter side. They do exactly the reverse process of what is done by the individual blocks of the transmitter. The receiver side blocks are ordered asOFDM de-multiplexer, demodulator, de-interleaver, FEC decoder and de-randomizer each of which is described as:

1. OFDM De-multiplexer receives noise corrupted signal from the channel. Since the signals are received as sub-signals which are orthogonal to each other along with the cyclic prefixes, this block removes the cyclic prefix and converts the low rate sub-channels to the wideband channel. It is done by a digital signal processing unit called FFT block.

2. The demodulator demodulates the signal that comes to it from the OFDM de-multiplexer. It converts or maps the signal waveform into the sequence of bits from which the signal was created. For PSK 
modulator at transmitter similar PSK demodulator at receiver is used. The higher the order of modulation the higher is the probability of error in demodulation. The demodulator should work ideally Otherwise, there are chances that errors are introduced in this block as well.

3. De-Interleaver does the opposite process of what interleaver has done at the transmitter to get the original sequence of the transmitted data. Since at the transmitter bits are written in row fashion and read in column fashion, at receiver bits are written in column fashion and read in the row fashion. This is especially useful in fading channels to prevent the long sequence of continuous errors.

4. FEC Decoder is used to remove the redundancies that were added at the transmitter to get the same amount of bits as there were before transmission. It detects and corrects the errors that occur during the transmission of the signal. Different coding schemes have different error detecting and correcting capabilities. Some channel codes are better than the others. The decoding procedure also depends on the type of FEC code used at the transmitter which works on some definite decoding algorithm.

5. De-Randomizer is a block which gets the original order of the bits as transmitted. The haphazard changes made at the transmitter side are undone at the receiver side, so we get the same message transmitted from the source to be delivered to the destination.

\section{Results Discussion}

The results are given in the figures below, which make use of two different channels and these channels are analysed for each of the four FEC codes and the following modulations- BPSK, QPSK, 16-PSK, and 64-PSK. In Figure 2(a), BER analysis of WiMAX over Rayleigh and AWGN channel has been done based on $\mathrm{BCH}$ codes and using BPSK. It is seen that for a BER of $10^{-3}$, SNR required by AWGN channel is $6 \mathrm{~dB}$ and that by Rayleigh channel is $9.6 \mathrm{~dB}$. There is an SNR improvement of $3.6 \mathrm{~dB}$ in the case of AWGN channel. The BER analysis of WiMAX over Rayleigh and AWGN channel has been again done using QPSK modulation and $\mathrm{BCH}$ codes as shown in Figure 2(b), the figure demonstrates that to get a BER of $10^{-3}$, SNR needed by AWGN channel is $6.4 \mathrm{~dB}$ and by Rayleigh channel is $8.2 \mathrm{~dB}$. The comparison shows an SNR improvement of $1.8 \mathrm{~dB}$ in the case of AWGN channel over Rayleigh channel. Now taking a look at the next figure, BER versus SNR graph of WiMAX over Rayleigh and AWGN channel has been given using the same FEC code but this time, 16-PSK. It can be understood well from Figure 2(c) that to achieve an error rate of $10^{-3}$, AWGN channel must have an SNR of $11.2 \mathrm{~dB}$ and Rayleigh channel must have an SNR of $13.5 \mathrm{~dB}$. The difference in the SNR values of two channels is $2.3 \mathrm{~dB}$. In Figure 2(d), is given a graph of WiMAX over Rayleigh and AWGN channel using 64-PSK modulation and BCH codes. From this graph, it can be determined that for a BER of $10^{-3}$, SNR constraint for AWGN channel is $18.8 \mathrm{~dB}$ and that for Rayleigh channel is $20.4 \mathrm{~dB}$ exhibiting an SNR improvement of $1.6 \mathrm{~dB}$ in the case of AWGN channel. Taking a look at the other figure of BER analysis of WiMAX system taken over Rayleigh and AWGN channel, the channel code used here is turbo code and modulation used is BPSK. It is seen that to achieve a bit error rate of $10^{-3}$, AWGN channel is supposed to have an SNR of $7.1 \mathrm{~dB}$ and Rayleigh channel must have an SNR of $8.8 \mathrm{~dB}$. This means that AWGN requires $1.7 \mathrm{~dB}$ lesser SNR for the same BER compared to Rayleigh channel and hence performs better as shown in Figure 3(a). Similarly, another graph for BER analysis of WiMAX over Rayleigh and AWGN channel is presented in Figure 3(b), using QPSK and along with Turbo codes. It can be well judged that for a BER of $10^{-3}$, SNR needed for AWGN channel is $7.1 \mathrm{~dB}$ and that for Rayleigh channel is $8.9 \mathrm{~dB}$, having improved by $1.8 \mathrm{~dB}$ in the case of AWGN channel. While in next figure named Figure 3(c), BER computation on SNR of WiMAX over Rayleigh and AWGN channel is taken using 16-PSK. The channel code used is again Turbo code. For achieving a BER of $10^{-3}$, SNR needed by AWGN channel is $11 \mathrm{~dB}$ and by Rayleigh channel is $12.8 \mathrm{~dB}$. There is again an SNR improvement of $1.8 \mathrm{~dB}$ in the case of AWGN channel for turbo codes. Over again, analysis of WiMAX over Rayleigh and AWGN channel has been done using Turbo codes, but modulation order is changed to 6 as given in Figure 3(d). It can be examined that a BER of $10^{-3}$ can be achieved at an SNR of $18.4 \mathrm{~dB}$ in AWGN channel and an SNR of $20.2 \mathrm{~dB}$ in Rayleigh channel. Therefore presenting an SNR improvement of $1.8 \mathrm{~dB}$ in the case of AWGN channel. 


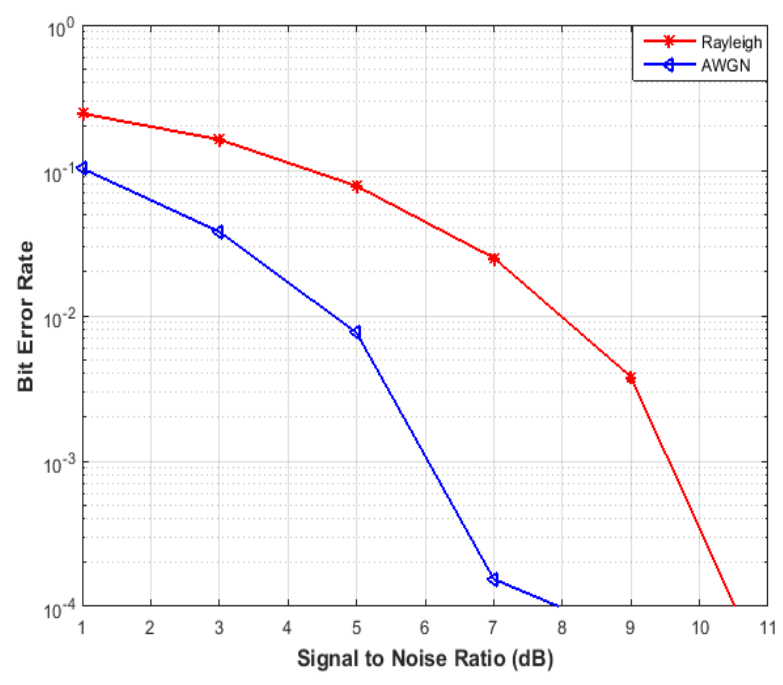

(a)

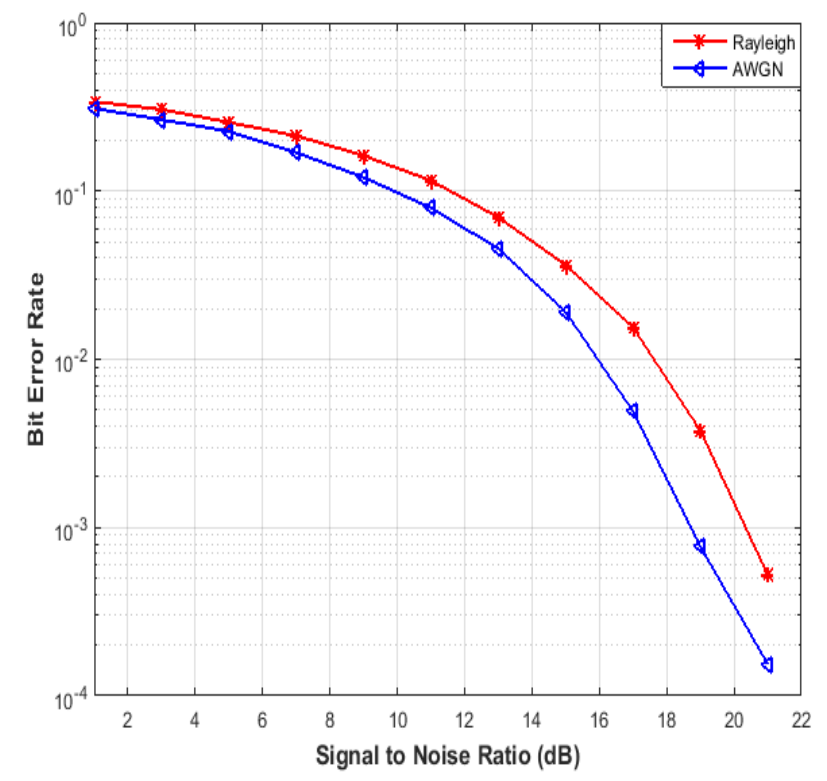

(c)

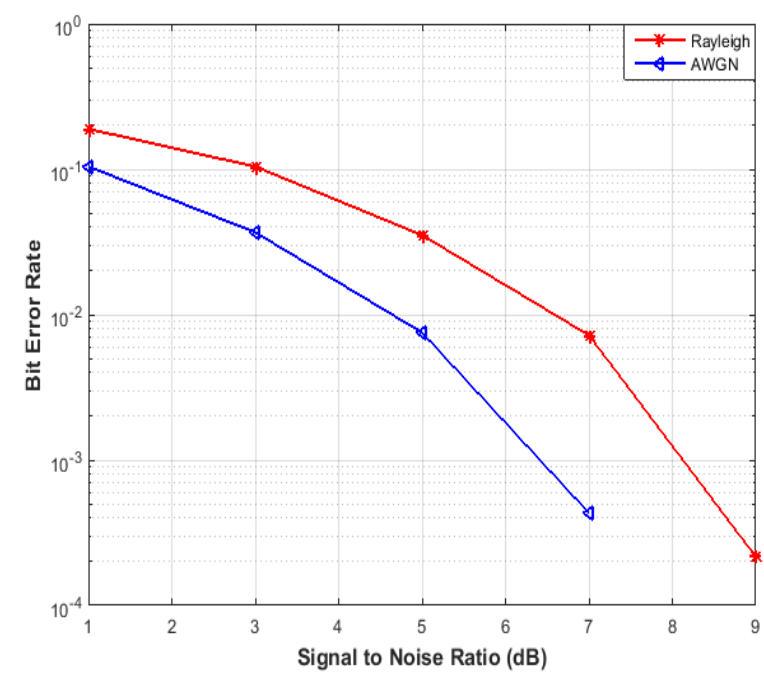

(b)

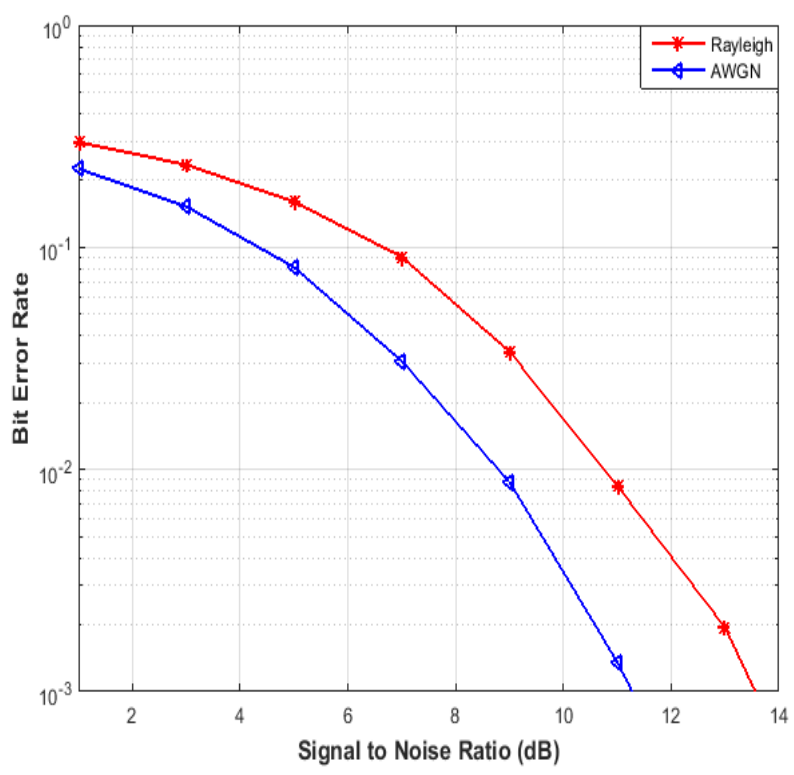

(d)

Figure 2 (a-d). SNR vs. BER Comparison for WiMAX based on BCH Codes over Rayleigh and AWGN Channels (a) BPSK (b) QPSK (c) 16-PSK (d) 64-PSK. 


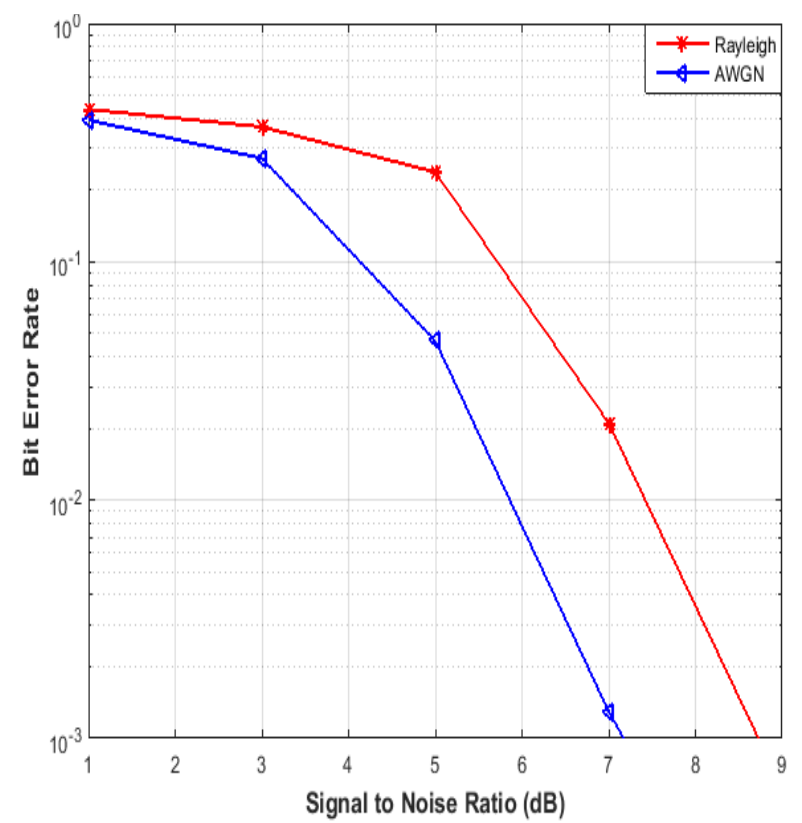

(a)

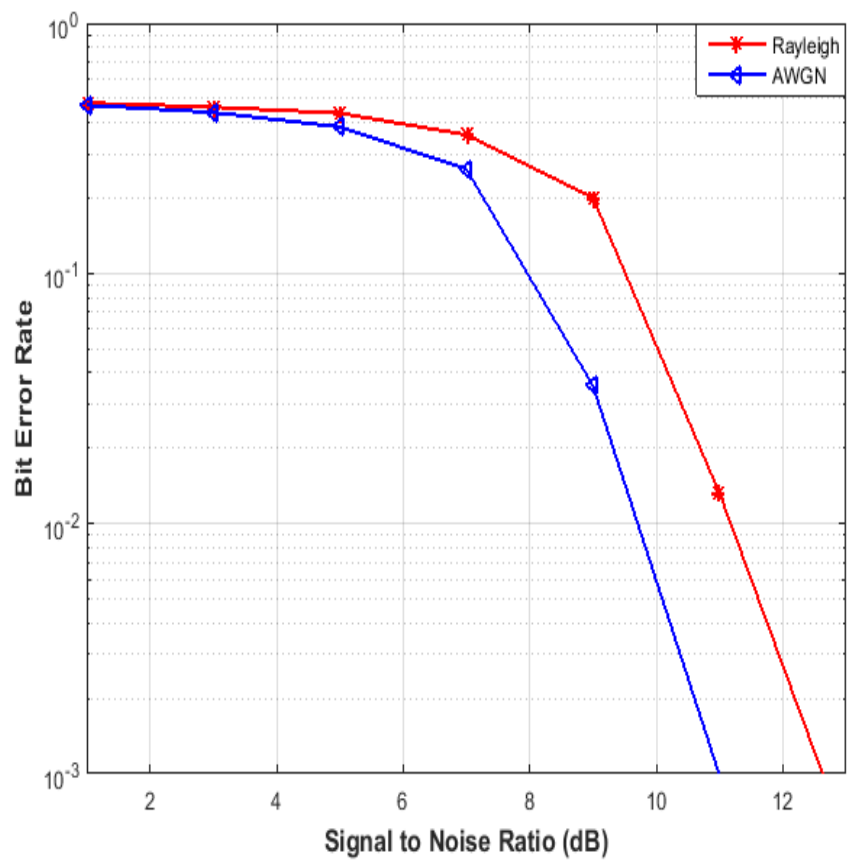

(c)

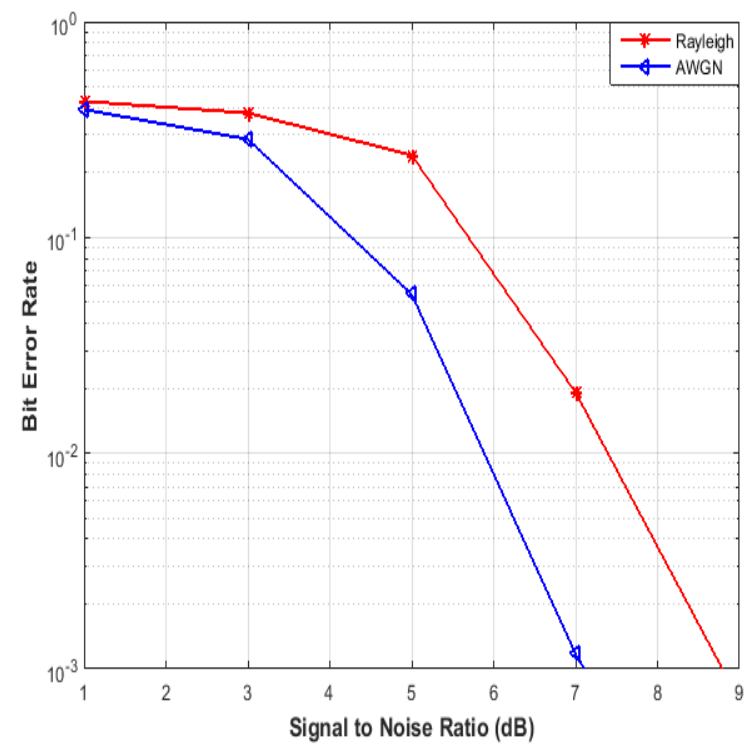

(b)

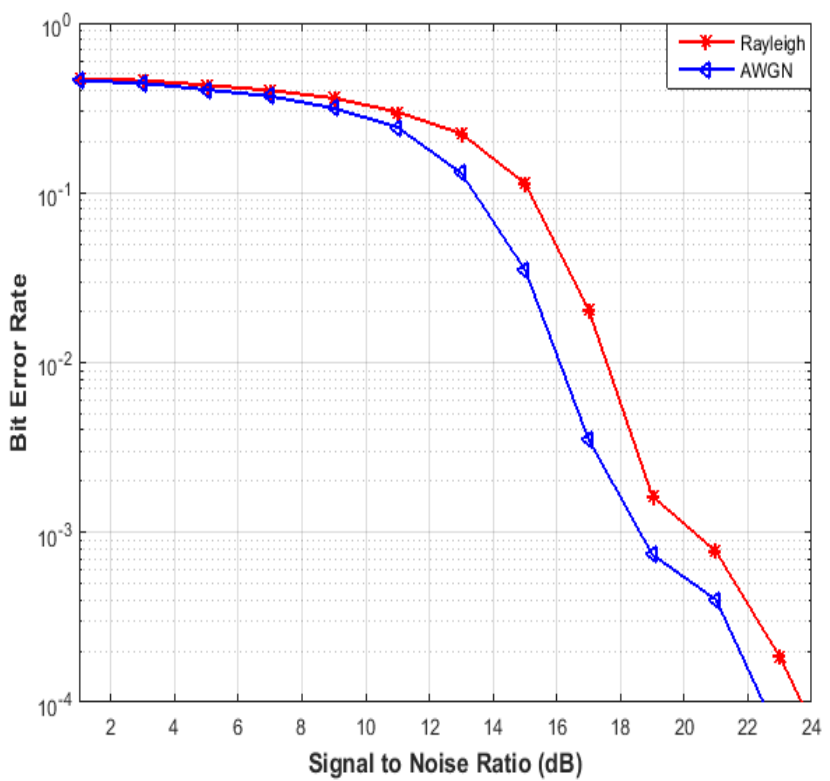

(d)

Figure 3 (a-d). SNR vs. BER Comparison for WiMAX based on Turbo Codes over Rayleigh and AWGN Channels (a) BPSK (b) QPSK (c) 16-PSK (d) 64-PSK. 


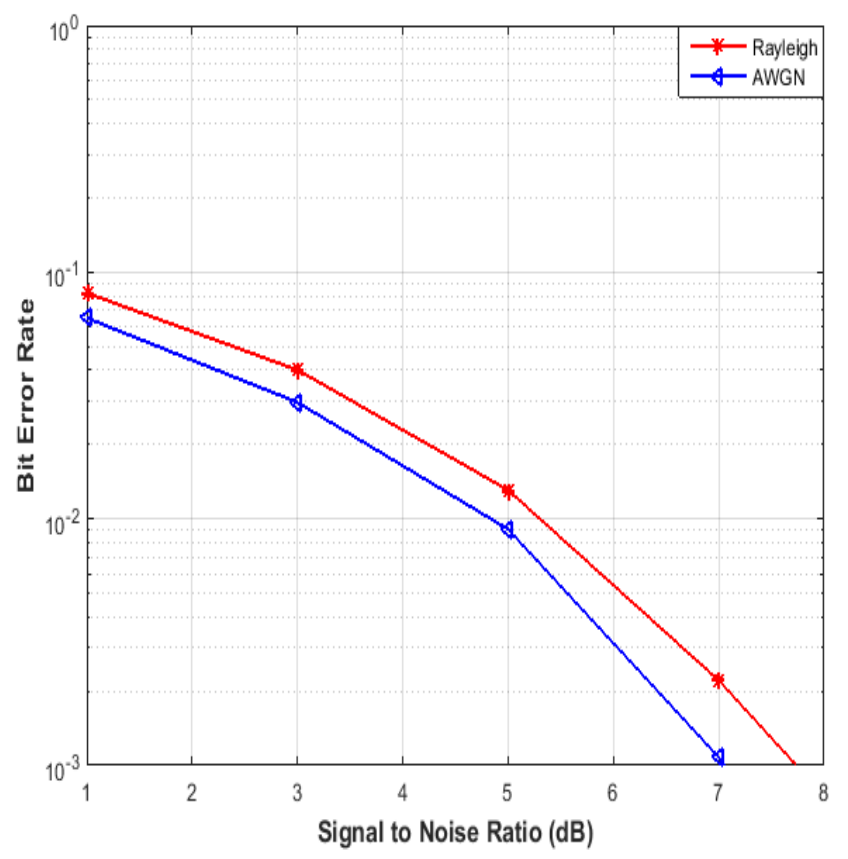

(a)

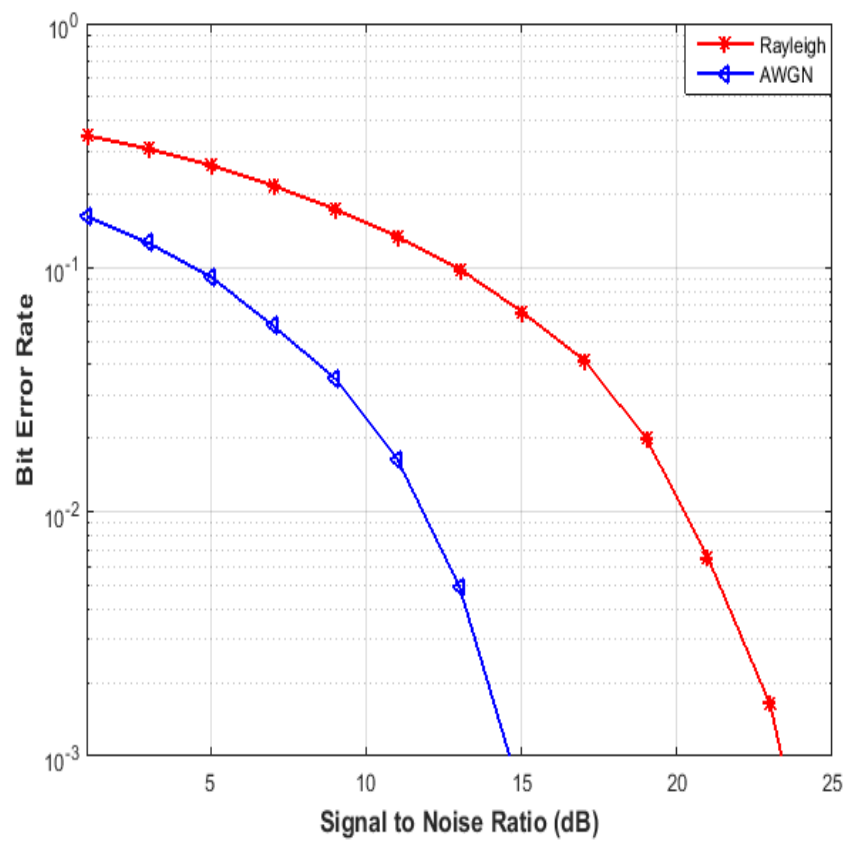

(c)

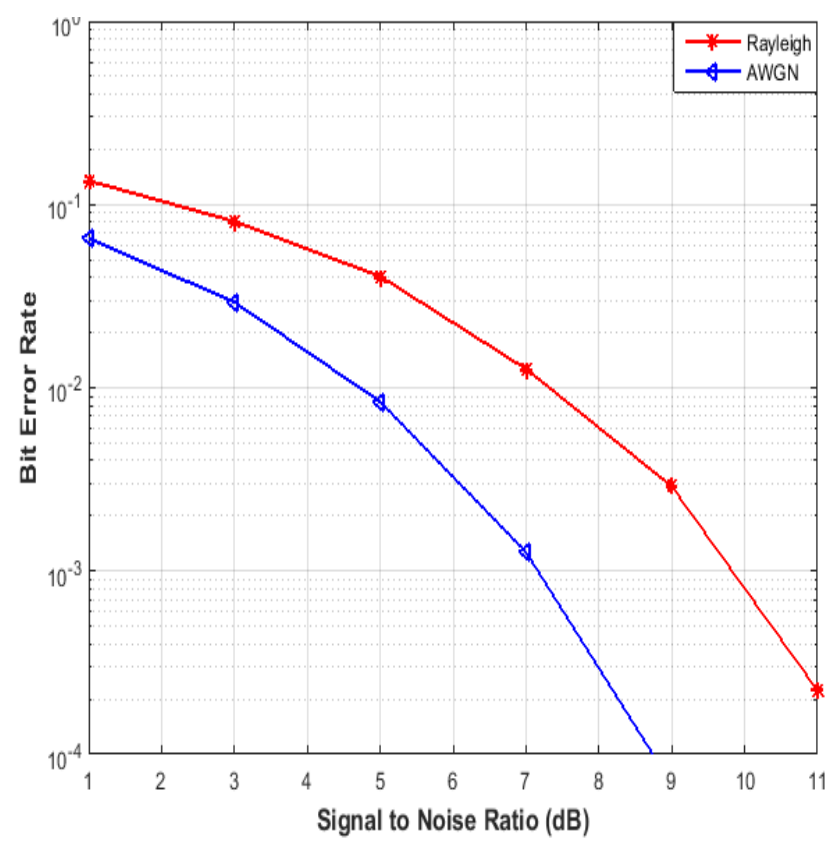

(b)

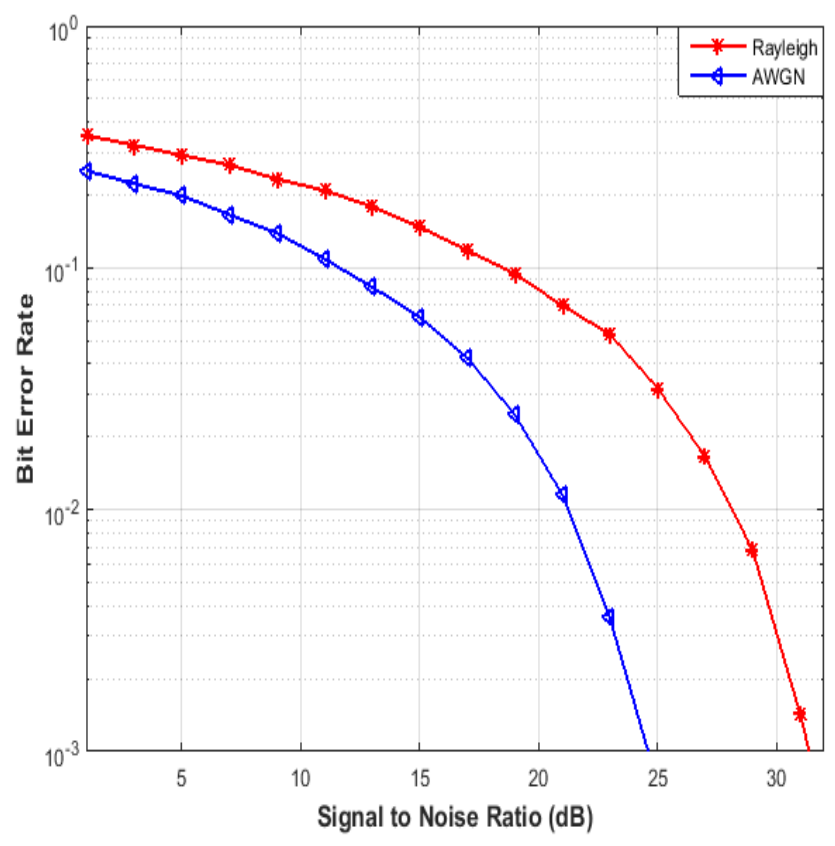

(d)

Figure 4 (a-d). SNR vs. BER Comparison for WiMAX based on LDPC Codes over Rayleigh and AWGN Channels (a) BPSK (b) QPSK (c) 16-PSK (d) 64-PSK. 


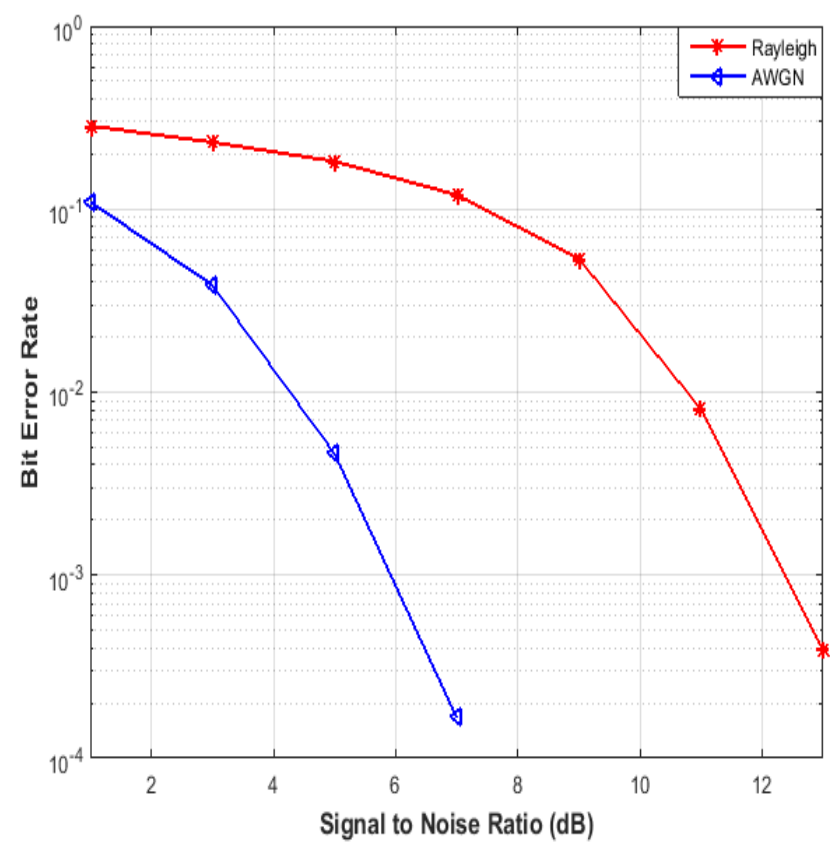

(a)

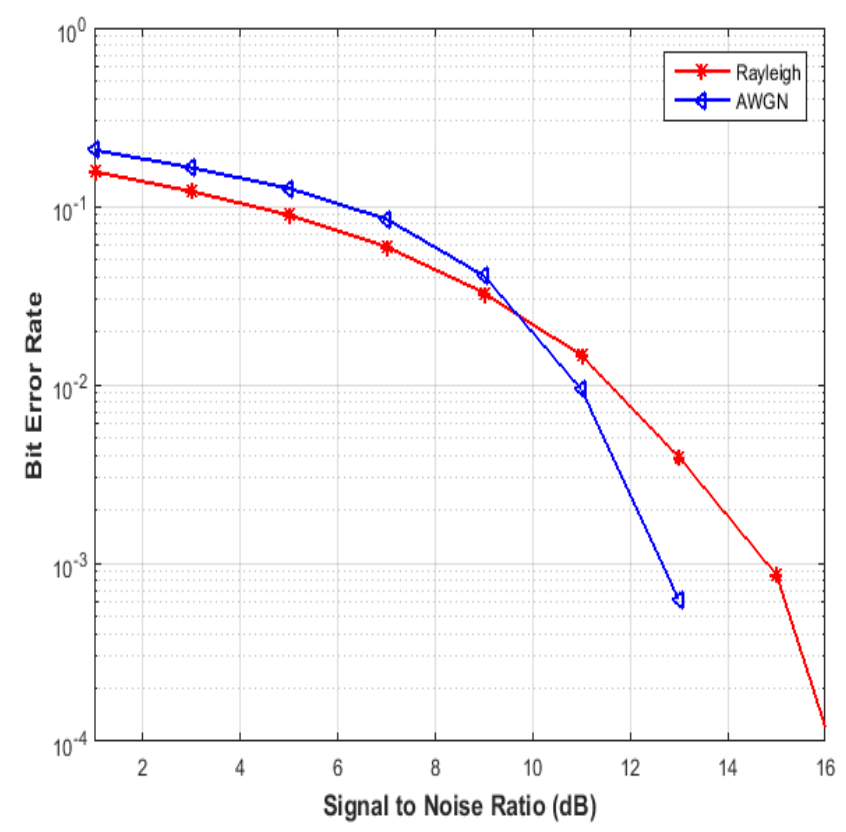

(c)

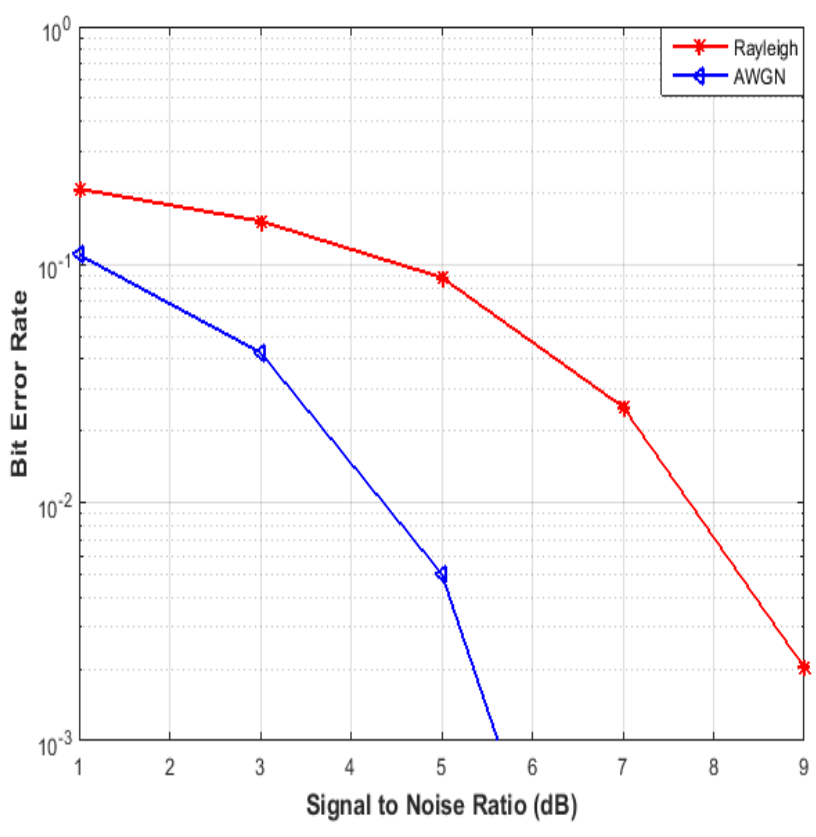

(b)

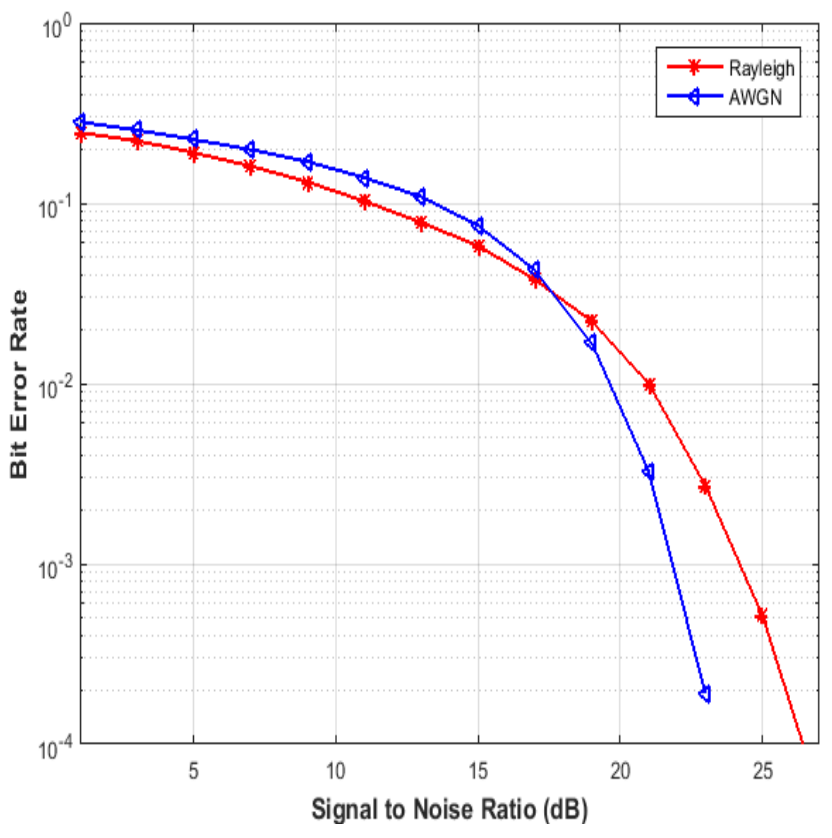

(d)

Figure 5 (a-d). SNR vs. BER Comparison for WiMAX based on RS Codes over Rayleigh and AWGN Channels (a) BPSK (b) QPSK (c) 16-PSK (d) 64-PSK. 
In Figure 4(a), BER analysis of WiMAX over Rayleigh and AWGN channel has been done based on LDPC codes and using BPSK. It is seen that for a BER of $10^{-3}$, SNR required by AWGN channel is $7 \mathrm{~dB}$ and that by Rayleigh channel is $7.7 \mathrm{~dB}$. There is an SNR improvement of $0.7 \mathrm{~dB}$ in the case of AWGN channel. The BER analysis of WiMAX over Rayleigh and AWGN channel has been again done using QPSK modulation and LDPC codes as shown in Figure 4(b), the figure demonstrates that to get a BER of $10^{-3}$, SNR needed by AWGN channel is $7.1 \mathrm{~dB}$ and by Rayleigh channel is $9.8 \mathrm{~dB}$. The comparison shows an SNR improvement of $2.7 \mathrm{~dB}$ in the case of AWGN channel over Rayleigh channel. Now taking a look at the next figure, BER versus SNR graph of WiMAX over Rayleigh and AWGN channel has been given using the same FEC code but this time, 16-PSK. It can be understood well from Figure 4(c) that to achieve an error rate of $10^{-3}$, AWGN channel must have an SNR of $14.5 \mathrm{~dB}$ and Rayleigh channel must have an SNR of $23.5 \mathrm{~dB}$. The difference in the SNR values of two channels is 9dB. In Figure 4(d), a graph of WiMAX over Rayleigh and AWGN channel using 64-PSK modulation and LDPC codes is given. From this graph, it is seen that for a BER of $10^{-3}$, SNR constraint for AWGN channel is $24.5 \mathrm{~dB}$ and that for Rayleigh channel is $32.5 \mathrm{~dB}$ exhibiting an SNR improvement of $8 \mathrm{~dB}$ in the case of AWGN channel. Viewing the next figure of BER analysis of WiMAX system taken over Rayleigh and AWGN channel, the channel code used here is RS code and modulation used is BPSK. It is seen that to achieve a bit error rate of $10^{-3}$, AWGN channel is supposed to have an SNR of 5.9dB and Rayleigh channel must have an SNR of $12.5 \mathrm{~dB}$. This means that AWGN requires $6.6 \mathrm{~dB}$ lesser SNR for the same BER compared to Rayleigh channel and hence performs better as shown in Figure 5(a). Similarly, BER analysis of WiMAX over Rayleigh and AWGN channel is presented in Figure 5(b), using QPSK and along with RS codes. It can be well judged that for a BER of $10^{-3}$, SNR needed for AWGN channel is $5.6 \mathrm{~dB}$ and that for Rayleigh channel is $9 \mathrm{~dB}$, having improved by $3.4 \mathrm{~dB}$ in the case of AWGN channel. While in next figure named Figure 5(c), BER analysis of WiMAX over Rayleigh and AWGN channel using 16-PSK is shown where channel codes used is again RS codes. For achieving a BER of $10^{-3}$, SNR needed by AWGN channel is $12.8 \mathrm{~dB}$ and by Rayleigh channel is $15 \mathrm{~dB}$. There is an SNR improvement of $2.2 \mathrm{~dB}$ in the case of AWGN channel for RS codes. Again, analysis of WiMAX over Rayleigh and AWGN channel has been done using RS codes, but modulation order is changed to 6 as given in Figure 5(d). It can be examined that a BER of $10^{-3}$ can be achieved at an SNR of $23 \mathrm{~dB}$ in AWGN channel and an SNR of $27 \mathrm{~dB}$ in Rayleigh channel. Therefore presenting an SNR improvement of $9 \mathrm{~dB}$ in the case of AWGN channel. The result summary is given in the Table 1.

Table 1. SNR requirement in WiMAX for diverse channel encoding schemes

\begin{tabular}{|l|c|c|c|c|c|c|c|c|}
\hline \multirow{2}{*}{$\begin{array}{l}\text { Modu- } \\
\text { lation }\end{array}$} & \multicolumn{5}{|c|}{ SNR(dB) required to achieve a BER of $1^{-3}$} \\
\cline { 2 - 10 } & \multicolumn{3}{|c|}{ AWGN Channel } & \multicolumn{4}{|c|}{ Rayleigh Channel } \\
\cline { 2 - 9 } & BCH & LDPC & RS & TURBO & BCH & LDPC & RS & TURBO \\
\hline BPSK & 6 & 7 & 5.9 & 7.1 & 9.6 & 7.7 & 12.5 & 8.8 \\
\hline QPSK & 6.4 & 7.1 & 5.6 & 7.1 & 8.2 & 9.8 & 9 & 8.9 \\
\hline 16-PSK & 11.2 & 14.5 & 12.8 & 11 & 13.5 & 23.5 & 15 & 12.8 \\
\hline 64-PSK & 18.8 & 24.5 & 23 & 18.4 & 20.4 & 32.5 & 27 & 20.2 \\
\hline
\end{tabular}

\section{Conclusion}

The results discussed help us to conclude that AWGN channel performs much better than Rayleigh channel in any of the forward error correction codes used or modulation technique used. This is because the sole purpose of AWGN channel is to add white Gaussian noise to the signal passing through the channel whereas in case of Rayleigh channel there are many obstructions in the path of source and destination which leads to multipath fading, and there is no line of sight component from source to destination in Rayleigh fading channel. In the case of WiMAX based on BCH codes and various PSK modulation orders, there is an improvement of about $1.6 \mathrm{~dB}$ to $3.6 \mathrm{~dB}$ of $\mathrm{SNR}$ for the same value of BER. If the WiMAX system based on Turbo codes is considered than there is a constant SNR improvement of about $1.8 \mathrm{dBs}$ in any of the modulation order of PSK. Getting on to a system based on LDPC codes there is a wide range of improvement in SNR ranging from $0.7 \mathrm{~dB}$ to $9 \mathrm{dBs}$ for various modulations. In the end, taking a glance at the RS codes based WiMAX system the SNR improvement ranges between $2.2 \mathrm{dBs}$ to $6.6 \mathrm{dBs}$. Hence, the signal in Rayleigh channel fades drastically giving poor results as compared to AWGN channel. With wireless broadband systems like WiMAX, it is, therefore, preferable to have a channel resembling AWGN channel in its statistics and characteristics rather than a fading channel like Rayleigh channel. 


\section{References}

1. Ohrtman F, Mc Graw Hill. WiMAX Handbook 802.16 Wireless Networks; 2005.

2. Rappaport T. S. Wireless communications principles and practice. Second Edition, Pretence Hall; 2002.

3. Mohan K. J, Cheriyan R. High-Speed address generating design for the dynamic modulation scheme supportive WiMAX deinterleaver. In Proceedings of International Conference on Magnetics, Machines and Drives. Kottayam. Jul 2014. p. 1-5.

4. Xinyu Z, Xian. A basic research on forward error correction. In Proceedings of International Conference on Communication Software and Networks. May 2011. p. 462-65.

5. Ding C, Du X, Zhou Z. The bose and minimum distance of a class of $\mathrm{BCH}$ Codes. IEEE Transaction Information Theory. May 2015; 61(5):2351-356.

6. Campbell RL, Ebel WJ. Simulation issues for binary digital communication systems using $\mathrm{BCH}$ and $\mathrm{RS}$ codes. In Proceedings of IEEE, Bringing Together Science and Technology, Tampa, Florida, USA. April 1996. p. 191-94.
7. Almoliki YM, Aldhaeebi MA, Almwald GA, Shoabi MA. The performance of RS and RSCC coded cooperation system using higher order modulation schemes. In Proceedings of International Conference on Intelligent Systems, Modelling and Simulation, Kuala Lumpur. Feb2015. p. 211-14.

8. Liu H, Huang Q, Deng G. Chen J. Quasi-Cyclic representation and vector representation of RS-LDPC codes. IEEE Transactions on Communication. Feb 2015; 63 (4): 1033-42.

9. Patidar M, Dubey R, Jain NK, Kulpariya S. Performance analysis of WiMAX 802.16e Physical Layer. In Proceedings of S. Conference on Wireless and Optical Communication Networks, Indore. Sep 2012. p. 1-4.

10. Khan S, Fisal N, Bakar ZA, Salawu N, Maqbool W, Ullah $\mathrm{R}$, Safdar H. Secure authentication and key management protocols for mobile multihop WiMAX networks. Indian Journal of Science and Technology. Jan 2014; 7(3):282-95.

11. Singh C, Patterh MS, Sharma S. Design of programmable digital down converter for Wimax. Indian Journal of Science and Technology. Mar 2009; 2(3):20-21.

12. Qamar RA, Maarof MA, Ibrahim S. An efficient encoding algorithm for (n, k) binary cyclic codes. Indian Journal of Science and Technology. May 2012; 5(5):2757-61. 